Revue Sciences/Lettres

Sciences / Lettres

1 | 2013

Transferts culturels

\title{
Les archives de la création à l'âge du tout numérique
}

Pierre-Marc de Biasi

\section{(2) OpenEdition}

Journals

Édition électronique

URL : http://journals.openedition.org/rsl/314

DOI : $10.4000 /$ rsl. 314

ISSN : 2271-6246

Éditeur

Éditions Rue d'Ulm

Référence électronique

Pierre-Marc de Biasi, «Les archives de la création à l'âge du tout numérique », Revue Sciences/Lettres [En ligne], 1 | 2013, mis en ligne le 01 mai 2012, consulté le 05 mai 2019. URL : http:// journals.openedition.org/rsl/314; DOI : 10.4000/rsl.314

Ce document a été généré automatiquement le 5 mai 2019.

(c) Revue Sciences/Lettres 


\title{
Les archives de la création à l'âge du tout numérique
}

\author{
Pierre-Marc de Biasi
}

L'analyse et l'interprétation des archives de la création est devenue indissociable d'une discipline récente - la génétique des artéfacts - qui s'est fixé pour objectif de donner toute leur place aux documents de genèse dans l'étude des processus créatifs et l'approche critique des œuvres artistiques, littéraires, techniques et scientifiques. Dans le domaine de la littérature, où cette discipline s'est fondée ${ }^{1}$, la "génétique des textes » se propose de renouveler la connaissance des œuvres à la lumière de leurs manuscrits en déplaçant l'interrogation critique de l'auteur vers l'écrivain, de l'écrit vers l'écriture, de la structure vers les processus, de l'œuvre vers sa genèse. Le principe de ce déplacement relève de l'évidence : le texte définitif d'une œuvre est toujours le résultat d'un travail et d'une élaboration progressive, l'aboutissement d'une durée créative au cours de laquelle l'écrivain s'est consacré à la recherche de documents ou d'informations, à la conception, à la structuration et à la rédaction de son texte, à travers diverses campagnes de corrections et de révision qui ont fait se succéder un nombre parfois impressionnant de versions. La génétique se consacre donc résolument à cette dimension temporelle de l' avant texte et du devenir cuvre, en posant l'hypothèse que l'œuvre, dans sa version finale, reste l'effet des métamorphoses qui lui ont donné forme et qui se trouvent totalisées en elle comme la mémoire active de sa propre genèse.

2 Mais, pour pouvoir devenir l'objet d'une véritable étude, cette genèse de l'œuvre doit avoir laissé des «traces». Ce sont ces indices matériels que la génétique textuelle se propose de retrouver et de rendre intelligibles : «les manuscrits de travail» de l'œuvre (carnets, plans, notes, brouillons, copies, épreuves corrigées, etc.), souvent couverts de ratures, très variables en quantités et en types, que les écrivains nous ont transmis au cours des trois derniers siècles. Ils se comptent par centaines de millions de documents, mais restent encore aujourd'hui très largement inédits et inexplorés dans les collections patrimoniales. 


\section{Le manuscrit moderne : un transfert historique}

3 Ces autographes de travail sur lesquels travaille la critique génétique ne nous ont pas été rendus accessibles par l'effet du hasard: nous ne les possédons que parce que leurs auteurs, en premier lieu, ont pris soin de les conserver, de les sauvegarder et de nous les transmettre et, en second lieu, parce qu'une longue chaîne de transfert matériel et symbolique - celle du patrimoine et des bibliothèques - s'est progressivement constituée pour les protéger, les maintenir intacts et les cataloguer jusqu'à pouvoir aujourd'hui les mettre à notre disposition.

Ces «manuscrits modernes " se distinguent essentiellement des "manuscrits anciens » (antiques et médiévaux) que la philologie classique s'était donnés comme objets d'étude, et qui avaient assuré, avant l'imprimerie à caractères mobiles, le rôle de support quasiexclusif pour l'enregistrement, la communication et la diffusion publique des textes, notamment littéraires. Du XVI ${ }^{e}$ au $\mathrm{XVIII}^{\mathrm{e}}$ siècle, le manuscrit, en perdant progressivement son rôle éditorial, s'enrichit d'une nouvelle signification fortement valorisée : écrit « de la main de l'auteur ", il devient la trace d'une création individuelle, le témoin matériel et la signature d'une pensée qui est à l'origine du texte imprimé. À travers les papiers autographes, ce que l'on commence à ressentir comme le véritable enjeu du manuscrit, c'est la personne de l'écrivain, son travail, sa démarche, son individualité.

Cette promotion du manuscrit s'inscrit dans une révolution intellectuelle qui a construit notre modernité. Elle accompagne, dans l'Europe des Lumières, le développement d'une idéologie du sujet qui culmine chez Kant avec la théorie du " génie » et de l'« originalité ", mais aussi la constitution d'une science du devenir historique où la notion de «travail humain » acquiert, avec Hegel, une position centrale comme médiatisation du devenir. Le manuscrit autographe se trouve valorisé par la reconnaissance progressive des notions de "droit moral », de "propriété individuelle » et de "valeur intellectuelle », qui finissent par s'inscrire juridiquement en termes de «contrat d'édition », de "droits d'auteur » et de "propriété littéraire» (1793 et 1825-1826). Enfin, avec la révolution industrielle, l'émergence d'un clivage entre "produit» (objet anonyme de la mécanisation) et « œuvre » (objet personnel de l'artiste ou de l'artisan) et les débats sur « l'art industriel » (ancêtre de notre "industrie culturelle») se traduisent par une curiosité nouvelle du public cultivé pour le savoir-faire, le geste créatif où le sujet inscrit sa marque, selon une évolution qui mènera de la valorisation de l'originalité à la théorisation de l'autonomie artistique.

6 Ces déterminations se traduisent, entre le milieu du XVIII ${ }^{e}$ siècle et la fin du XIX siècle, par un phénomène sans précédent : les écrivains et les créateurs changent d'attitude à l'égard de leurs propres documents de travail. Au lieu de jeter leurs papiers après publication, ils commencent à conserver les archives de leur création en leur attribuant une valeur symbolique qui les rend dignes d'être transmises à la postérité, au même titre que l'œuvre publiée elle-même. En France, c'est avec Victor Hugo que ce nouveau rapport au manuscrit devient explicite par un geste qui va institutionnaliser l'édification d'un nouveau patrimoine écrit. En 1881, rédigeant un Codicille à son testament, il écrit : «Je donne tous mes manuscrits et tout ce qui sera trouvé écrit ou dessiné par moi à la bibliothèque nationale de Paris qui sera un jour la Bibliothèque des États-Unis d'Europe. » À partir de cette date, le principe même d'un transfert historique des documents de genèse est acquis : en léguant leurs dossiers autographes, les écrivains, les artistes et les 
savants, leurs ayants droit et leurs descendants, vont progressivement constituer un immense domaine documentaire disponible pour la recherche. Ce sont ces traces de la création que la génétique des textes, un siècle plus tard, s'est employée à redécouvrir et à valoriser, notamment, pour le domaine littéraire, en enrichissant l'étude du « texte » par l'exploration scientifique des processus dont il est l'effet: une valorisation qui n'a pu s'accomplir qu'avec l'aide et la contribution des grandes institutions de conservation, mais qui s'est aussi traduite par l'élaboration d'une théorie et d'une technique d'analyse spécifiques, requises pour le traitement de ces objets récalcitrants et textuellement incorrects que sont les documents de genèse. Rebuts de l'écriture, restes pléthoriques traversés par la disgrâce de l'approximatif et le démon de l'inachevé, réputés d'une opacité impénétrable en raison de leurs innombrables ratures, les «manuscrits de travail » que la génétique a déversés par tombereaux sur la scène critique n'ont pas toujours été accueillis chaleureusement par le milieu académique, et il y avait de quoi.

Conservés dans un état illisible et ininterprétable, parce que non classés, non déchiffrés, et parfois non encore inventoriés, ces indices génétiques ont le désavantage de ne pas être intelligibles sans un important travail préliminaire qui peut être considéré comme rebutant ou dissuasif. Mais la réciproque de cette difficulté n'est pas non plus négligeable, car ces archives sont aussi celles qui, moyennant un peu de patience, peuvent conduire le critique à un trésor de connaissances de première main, aussi inédites qu'irremplaçables : l'histoire de ce qui s'est passé entre le moment où l'écrivain a commencé à entrevoir la première idée de son projet et le moment où le texte, écrit et corrigé, paraît sous la forme d'un livre imprimé. En supposant que ces documents contiennent des informations inédites et de première main sur l'œuvre, la génétique textuelle (qui analyse les manuscrits : les classe, les déchiffre et transcrit) et la critique génétique (qui interprète les résultats de cette analyse) cherchent à reconstituer l'avant-texte de l'œuvre (les phases successives du «texte à l'état naissant » et de sa fabrication) avec l'objectif d'élucider son sens et sa portée d'après ses processus de conception et de rédaction.

\section{L'approche numérique des documents de genèse}

8 Les liens originaires entre génétique des textes et environnement numérique sont nombreux. Le manuscrit moderne des trois derniers siècles $\left(\mathrm{xVIII}^{\mathrm{e}}-\mathrm{XX}^{\mathrm{e}}\right)$ est un artéfact de papier, dont l'analyse scientifique s'est tout de suite trouvée liée à l'informatique. Même si cette complicité ne s'est révélée qu'avec le temps, les dates restent éloquentes. Le terme de "génétique " appliqué à l'étude des processus d'écriture, le développement d'une approche concertée sur plusieurs grands corpus littéraires tout comme la fondation de la structure de recherche elle-même (CAM 1975-1981, ITEM depuis 1982) coïncident précisément avec la période qui voit émerger l'informatique conviviale. Cette coïncidence contient un symbole: celui d'un lien mélancolique entre la science et son objet. L'ethnologie a pris son essor quand il est devenu clair que les dernières cultures primitives étaient condamnées à disparaître. L'écologie et la climatologie se sont acquis toutes nos faveurs depuis que la planète a vu se désintégrer les équilibres qui assuraient sa survie. L'intérêt scientifique pour les manuscrits modernes a émergé quand s'est généralisé l'instrument qui annonçait leur prochaine disparition.

Il y avait eu des précédents. À l'époque des cartes perforées, quand les ordinateurs avaient encore la taille d'un F3, quelques généticiens de la première heure s'étaient attelés à des investigations un peu folles, d'une manière qui prend aujourd'hui une allure 
visionnaire. Mais, en pratique, c'est bien la révolution numérique des années 1980-1990, le traitement de texte, les bases de données et la montée en puissance d'Internet qui ont redéfini entièrement le champ et les outils des études de genèse en apportant des réponses aux questions logiques et quantitatives qui restaient jusque là insolubles.

Insolubles d'abord par la dimension des corpus, en vertu de cette disproportion qui caractérise l'archive génétique, souvent dix fois plus volumineuse que le texte lui-même. Comment éditer les manuscrits de travail d'un roman comme L'Éducation sentimentale de Gustave Flaubert, qui totalisent cinq mille pages de dimension A3 et des centaines de pages de carnets? Converti en livres respectant les dimensions des manuscrits originaux, l'ensemble des fac-similés et des transcriptions se traduirait par l'équivalent de dix à douze volumes in-quarto ou in-folio de mille pages, le double en format A4. Aucun éditeur doué de raison ne s'engagerait dans un pari si risqué2. Ces soucis quantitatifs sont presque inexistants pour le médium numérique, dont les outils gagnent en capacité de mémoire chaque année, et qui se combine en outre spontanément avec les capacités de diffusion propre au réseau Internet.

11 Mais, plus encore que le quantitatif, c'est pour ses capacités logiques que le médium numérique s'est vite révélé indispensable aux recherches en génétique. Formé de pages reliées les unes derrière les autres dans l'ordre de sa pagination, le livre est séquentiel : sa structure est plus que mal adaptée pour rendre raison des itinéraires complexes, mouvants et non linéaires qui sont ceux de l'écriture ou du work in progress. Où classer la transcription d'un folio qui comporte des notes écrites à plusieurs mois ou années de distance ou plusieurs fragments de brouillons qui se rapportent à des secteurs différents de l'œuvre? Comment mettre en scène dans un livre la manière dont l'écrivain mobilise simultanément six ou sept documents? Le cas est banal : par exemple, l'écrivain consulte le plan-scénario qui guide sa rédaction, l'état rédactionnel antérieur du fragment qu'il est en train de réécrire, un carnet où se trouvent des notes prises pour ce fragment, un plan de ville ou une carte, des coupures de journaux et peut-être un ou deux livres, ouverts aux pages où il a repéré des passages qui pourraient informer son écriture... L'outil informatique, qui permet de définir et d'indexer des blocs et des liens en nombre indéfini, semble bien adapté à ce contexte à la fois éclaté et synthétique, quels que soient sa complexité et le nombre des éléments mis en jeu. À cette échelle, il s'agit de rendre intelligible la synergie d'un environnement de travail à composantes multiples : par exemple, la table de l'écrivain où une page s'écrit en prélevant des contenus ou des consignes dans plusieurs sources à la fois. Le livre ne le pouvait, très laborieusement, qu'au prix de tableaux à doubles entrées, de lourdes digressions et de mises en pages dignes de la Kabbale. L'environnement numérique offre toute une gamme de scénographies possibles pour en traduire la dynamique au plus près des processus qui se trouvent en jeu.

En technique comme en théorie, cette avancée dans l'ère du " tout numérique » n'a donc pas été un obstacle, mais au contraire une condition très favorable au traitement scientifique de l'indice génétique. On peut même dire que cette mutation technique a été la condition sine qua non de l'émergence de la génétique : l'étude des archives modernes, celles des trois derniers siècles, n'est devenue réellement envisageable, surtout pour les grands corpus et les documents très complexes, que sous l'effet de cette révolution. L'outil informatique a été décisif et il continue de l'être, notamment pour la communication et la transmission des résultats : c'est dans la logique des éditions en ligne XML et des normes de type TEI que les généticiens de l'ITEM travaillent aujourd'hui à 
mettre au point un environnement de transcription et d'édition à vocation universelle pour les manuscrits de travail. C'est aussi, pour une large part, grâce aux énormes capacités du numérique qu'il est devenu possible d'envisager une extension des recherches sur les processus de création aux domaines non littéraires (l'écriture scientifique, ou le texte philosophique) ou même non textuels, comme c'est le cas, en "génétique des formes", pour les archives musicales, l'histoire de l'art (projets des architectes, esquisses et repentirs des plasticiens), ou les arts visuels (dossiers de genèses filmiques ou photographiques). Dans tous ces champs d'investigation, c'est l'informatique qui a permis d'envisager un travail d'analyse approfondi sur chaque document et une étude systématique des très grands fonds, notamment grâce à la conversion des documents papier en données et en images numériques susceptibles de traitements de masse. C'est d'ailleurs la seule solution envisageable lorsqu'il s'agit, comme en génétique filmique, d'associer, dans un même mouvement de genèse, des éléments par nature aussi hétérogènes que des textes ou des notes autographes, des voix, du bruitage, de la musique, et des rushes: ramenés à leur dénominateur numérique commun, tous ces éléments se trouvent spontanément prêts au dialogue ou à la comparaison critique dans une même machine. Un ban de montage numérique en donne la démonstration immédiate : qu'il s'agisse de textes, d'images fixes, de sons enregistrés, ou de séquences d'images, c'est avec les mêmes outils, les mêmes paramètres, les mêmes visualisations et le même écran que l'on sera conduit à mener l'analyse intégrée de tous les éléments, chaque mode d'expression se présentant comme une couche du même continuum temporel.

\section{L'archive à l'âge du tout numérique}

13 Tout va donc pour le mieux dans les relations de complicité natives entre informatique et analyse des archives de la création : l'impénétrable masse des documents papier produits et conservés depuis le $\mathrm{XVIII}^{\mathrm{e}}$ siècle devient manipulable et intelligible en se dotant de la légèreté «immatérielle » et des flexibilités logiques du numérique. Mais qu'en est-il si l'on considère les corpus génétiques de notre temps: les archives de ce qui se crée et s'invente aujourd'hui ? Car la génétique des textes et des formes entretient aussi, depuis sa fondation, une relation vitale avec l'actualité de la création : dans les années 1970-1980, c'est en partie sous l'impulsion des écrivains eux-mêmes (Sartre, Ponge et sa Fabrique du pré, Aragon qui lègue ses manuscrits au CNRS, etc.) que les chercheurs ont commencé à former les premières propositions méthodologiques de la discipline. Et cette tradition de dialogue avec la création vivante reste bien présente aujourd'hui. Or, dans nos relations de travail avec la BnF, l'IMEC et les collections patrimoniales, ou directement avec les écrivains, leurs ayants droit ou les nouveaux donateurs de fonds, que voit-on se profiler en matière d'évolution des archives? Les créateurs confirmés qui lèguent leurs dossiers de genèse des années 1960-2000 transmettent des documents dont les médiums sont ceux $\mathrm{du} \mathrm{xx}^{\mathrm{e}}$ siècle : papier, bande magnétique, film pellicule, photo argentique, avec parfois quelques éléments plus récents qui relèvent du traitement de texte et des supports électroniques. Mais pour les créateurs de la génération suivante, celle qui a commencé à produire dans les années 1980, et plus nettement encore pour les jeunes créateurs contemporains, ceux des années 2000, la situation est toute différente: ce sont leurs archives elles-mêmes qui sont majoritairement numériques. 

elle a bouleversé le présent et l'avenir de toute la production intellectuelle et artistique. Le peintre résiste avec sa toile et ses tubes de couleurs, l'écrivain avec son calepin, le sculpteur avec son burin ou son chalumeau, l'architecte avec son calque d'étude... mais pour l'essentiel le médium électronique s'est imposé à la majorité des gestes créatifs, aussi bien pour celui qui écrit un roman, que pour celui qui compose un opéra, dessine un bâtiment, ou réalise une fiction cinématographique... Sans parler, bien entendu, de l'omniprésence de l'outil informatique dans la création scientifique, technique et industrielle. Cette homogénéisation fonctionnelle de toutes les pratiques créatives sous la tutelle hégémonique d'un seul et même médium crée une situation qui n'a jamais eu d'équivalent dans l'Histoire. Pour la première fois depuis les origines de la culture, qu'il s'agisse du calcul ou de l'écriture, du son (naturel ou artificiel ; bande-son, musique, voix, bruitage), de l'image et des artéfacts visuels (fixes ou animés, enregistrés ou dessin, photo, cinéma), des représentations volumiques (maquette, simulations 3D, environnements virtuels augmentés), etc., les modes d'expression jusque-là distincts par leurs techniques, leurs supports et leur savoir-faire se trouvent désormais assujettis au même médium, aux mêmes machines, et in fine au même code binaire. Et cette tutelle sans dehors s'exerce dans une intégration d'autant plus profonde qu'elle affecte à la fois et simultanément les conditions de la création, les pratiques expressives et leurs outils, mais aussi, sous l'effet d'Internet, la circulation des savoirs, la communication avec les pairs, l'accessibilité aux sources et, finalement, la diffusion des œuvres, la relation au public.

L'écrivain d'aujourd'hui n'échappe pas à cette mutation. Dans sa vie quotidienne comme dans son travail, il a pris l'habitude d'écrire et de corriger directement à l'écran : plus de tirages d'imprimante, comme il le faisait encore il y a dix ans, pour se relire tranquillement et reprendre son texte à la main, avant de saisir les corrections au clavier. Plus de correspondance (juste des courriels, des sms et des coups de fil cellulaires, tous numériques) $)^{3}$, plus de manuscrits, plus de biffures. Les prophètes du bureau sans papier ont fini par gagner. Inter ou intra, maintenant tout est net. L'ère du parchemin avait été celle du palimpseste, l'âge du papier celui de la rature, voici venue l'ère du support sans repentir : l'écran où rien d'autre ne subsiste que le texte toujours déjà typographique, prêt à imprimer, ou mieux, prêt à consommer, tout frais, en ligne, à même son affichage lumineux. conclure : plus de manuscrits, plus de brouillons? c'est la fin de la génétique! Dommage pour les généticiens, les pauvres : les voilà condamnés à fermer boutique... Bien entendu, le raisonnement est bancal et la conclusion idiote: ce n'est pas parce qu'on écrit au clavier qu'on trouve tout de suite sa formulation définitive, et ce n'est pas parce que l'écran reste propre que les ratures ont disparu. L'évanouissement des repentirs est une simple commodité périphérique, leur disparition réelle n'est pas une fatalité. D'ailleurs, en serait-il même ainsi que la génétique, avec ses trois siècles de brouillons inédits à traiter, ne manquerait pas de grain à moudre pour les cent ans à venir... Donc, n'en déplaise aux impatients, il faudra encore faire avec la génétique pour un bon moment. Mais la question n'est évidemment pas là. La vraie question est de comprendre que nous nous trouvons réellement aujourd'hui devant un dilemme crucial et sans précédent, qui pourrait bien se solder par deux formes d'une même alternative, aussi solidaires que les deux faces d'une pièce de monnaie, "de singe", sans doute: tout perdre ou tout conserver, communiquer ou transmettre.

Revue Sciences/Lettres, 1 | 2013 


\section{Laporie des nouveaux supports}

\section{Tout conserver}

17 Ce n'est pas parce que l'écran reste toujours propre qu'il ne subsiste aucune trace des opérations d'écriture qui ont précédé. C'est même le contraire. Moyennant quelques précautions, dont la sauvegarde des données sur un support pérenne qui garantisse leur stabilité et leur lisibilité à long terme, l'ordinateur a la capacité de conserver spontanément la trace de toutes les commandes que vous lui avez adressées, toutes intégralement, d'un bout à l'autre de chaque session de travail, et bien au-delà, aussi longtemps que vous ne procéderez pas à un écrasement délibéré de sa mémoire. Même dans ce cas, d'ailleurs, à moins que vous ne détruisiez physiquement le support matériel lui-même, des procédures d'extraction au moins partielles resteront toujours possibles comme le démontre à l'envi, de la brigade financière à la brigade des mœurs, le succès des enquêtes policières au cœur des disques durs. À l'échelle d'une session de travail, ces traces sont rendues accessibles grâce à un outil qui équipe tous les traitements de texte et tous les logiciels de dessin assisté, depuis les origines: la sauvegarde automatique paramétrable. Une copie automatique indexera par exemple toutes les quinze secondes une mise à jour du texte que vous rédigez : aucun coût en mémoire, puisque c'est le même texte à quelques détails près. Pour des raisons d'ergonomie faciles à comprendre, l'habitude est de tenir pour la «meilleure » la version la plus récente, en « oubliant » les versions précédentes. Mais, pour un moment au moins, tout reste disponible: par la procédure "annuler la frappe ", vous remontez à rebours la chaîne des modifications auxquelles vous vous êtes livré, caractère par caractère, commande par commande, preuve indiscutable que c'est bien de l'écriture et non du texte que l'ordinateur enregistre et compile. Sur ce modèle ont été mis au point des logiciels expérimentaux permettant de faire réapparaître à l'écran les états successifs d'une rédaction: ils donnent à voir la concaténation temporelle des métamorphoses du texte, séquence par séquence, chaque état variant ayant été indexé et sauvegardé. Mis bout à bout, tous ces détails, qui racontent la genèse de votre texte à la seconde près, quel nom faut-il leur donner sinon celui de manuscrit numérique? Un manuscrit booléen à support silicium certes, mais assorti de toutes les caractéristiques de son prédécesseur sur cellulose: ajouts, substitutions, suppressions, déplacements, etc.

18 Conclusion? Vous êtes un grand écrivain et vous souhaitez léguer à la postérité les archives de votre création? Déposez votre disque dur à la BnF, à l'IMEC ou à votre bibliothèque municipale préférée. Ce sera mille fois moins encombrant pour les conservateurs, et infiniment plus commode pour les chercheurs, que les liasses de papiers de Flaubert, de Proust ou de Roland Barthes. Pour le généticien qui cherche à reconstituer les phases de votre travail, le disque dur, c'est l'olympe : tous vos gestes d'écriture s'y retrouvent, et qui plus est classés, répertoriés, horodatés au dixième de seconde près. Un vrai miracle quand on sait ce qu'il faut de patience et de foi presque cistercienne pour mettre en ordre les pages d'un manuscrit, pour reconstituer sa capricieuse chronologie, et pire encore, pour dater les campagnes de ratures qui prolifèrent sur chaque page. Sans parler des fichiers de recherches documentaires annexées aux rédactions: c'est aujourd'hui toute l'exogenèse qui à son tour est devenue numérique, en intégrant à l'intertexte encyclopédique et multilingue du réseau mondial les ressources illimitées des 
moteurs de recherche et les univers multimédias du son et de l'image. Sans oublier l'essentiel : à la différence de ses prédécesseurs en papier, ce dossier de genèse numérique constitue un tout homogène : il a toutes les chances d'être exhaustif. Fini le drame des fonds lacunaires où il manque les plus belles pièces du puzzle, achetées en sous-main par un négociant du Texas, un trader britannique ou un nouveau riche de Shanghai : avec le manuscrit numérique, aucun folio ne s'égare, toutes les traces sont bien à l'abri dans leur capsule électronique, prêtes à la duplication immédiate : le rêve !

\section{Un âge d'or de la genèse}

19 Et ce n'est pas tout. Il y a aussi et surtout la « boîte noire ». Si les chercheurs s'intéressent aux brouillons, c'est pour reconstituer un processus mental : pour comprendre comment s'est formée une œuvre, en remontant aussi loin qu'il est possible en amont dans l'avanttexte, en explorant les prémices de cette phase prérédactionnelle où le projet prend sa source. Que reste-t-il de cette délibération à l'âge de la feuille de papier et du stylo? Imaginons - comme c'est effectivement le cas pour de nombreuses œuvres littéraires qu'une image secrète, une idée indécente, un fantasme inavouable, fût à l'origine du projet. Les premiers mots jetés par l'écrivain sur la page blanche en porteront-ils témoignage? Parfois oui, mais le plus souvent non, car lorsqu'on écrit sur du papier, on sait bien qu'on exhibe une trace durable - scripta manent-, qui deviendra lisible par n'importe qui : ce qui est la dernière des choses à faire pour un secret. Même s'il ne s'agit pas d'un détail avilissant, mais d'une simple incertitude, il y aura autocensure. Que la phrase ne soit pas encore tout à fait stable, et la petite instance parlante qui pilote l'opération au fond du cerveau dira: non, ça, tu ne l'écriras pas sous cette forme car, de toute façon, il te faudrait aussitôt le corriger, et ce serait bête de commencer par une grosse rature sur ce feuillet vierge. D'où, la fameuse page qui reste blanche. Une phrase d'incipit formulée au premier jet sur papier présuppose la succession d'une vingtaine de simulations mentales. C'est évidemment beaucoup plus qu'il n'en faut pour trouver le moyen de cacher l'essentiel.

Maintenant, reprenons notre écrivain en train de chercher sa première phrase, non plus devant sa page blanche, mais à son clavier d'ordinateur. Ce n'est plus le même homme : il sait (il croit) que rien ne subsistera de ses maladresses ou de ses abominations, même s'il les jette à l'état brut sur son écran: d'un geste il pourra les faire disparaître et y substituer tout autre chose : ni vu ni connu, l'écran fait place nette. Inutile pour se lancer d'attendre que la phrase soit construite mentalement : elle finira toujours par trouver son équilibre sans laisser trace des expressions inachevées ou contrefaites qui lui ont donné naissance. Au lieu des vingt tests initiaux qu'exigeait le papier, l'écriture à l'écran ne supposera que quatre ou cinq simulations préliminaires. Et voilà pourquoi le brouillon numérique constitue un document d'une richesse cognitive sans précédent : il donne au généticien un accès à des processus psychiques beaucoup plus en amont que ceux dont le papier peut porter la trace. Une grande part de l'élaboration mentale ne s'y trouve pas, certes, surtout celle qui trouve ses sources dans le cycle long de la mémoire; et l'autocensure y garde ses droits, mais en donnant quand même à voir une zone jusqu'ici inconnue de l'écriture à l'état naissant. Il ne s'agit plus tout à fait de la même écriture non plus, puisque précisément, à la différence de ce qui advenait avec le papier, le scripteur efface la trace de ses ratures et de ses hésitations à mesure qu'il corrige. Mais c'est bien l'écriture de notre temps. 


\section{Vers une science des processus}

21 L'ère numérique ne sera donc pas la fin du brouillon, mais peut-être son véritable commencement, et sans doute son âge d'or. Les spécialistes de la genèse ne craignent nullement le chômage technique, ils s'inquiéteraient plutôt du contraire. Jusqu'ici l'approche génétique ne portait que sur des exceptions: des fonds miraculeusement épargnés de la destruction, pour chaque culture quelques centaines de corpus complets par siècle, tout au plus. Même en se limitant aux plus hautes productions de l'esprit, qu'adviendra-t-il lorsque nous posséderons l'intégralité de tous les brouillons dans le domaine des lettres, des arts, des sciences et des techniques? La miniaturisation des supports, la capacité grandissante des matériels et la passion pour les archives permettent de supposer que, si l'on y met le prix, tout désormais pourra se conserver sous un format ergonomique. Mais alors, comment interpréter cette masse infinie de métamorphoses? Même si ces gisements de l'avenir doivent contenir des trésors de pensée et d'innovation, aurons-nous simplement assez de chercheurs pour en extraire les secrets et pour élaborer une véritable science des processus? Il se pourrait bien que nous nous inquiétions à tort : numériques par nature, les documents de genèse d'aujourd'hui ont une structure spontanément prête à l'inventaire raisonné et au calcul. Ils attendent simplement les machines qui sauront nous aider à les rendre intelligibles et à les interpréter, quelle que soit la discipline à laquelle ils appartiennent.

Pour ces nouveaux corpus électroniques comme pour les gisements de savoir génétique qui sommeillent encore dans les archives papier de la pensée moderne et contemporaine, la révolution numérique constitue la promesse de découvertes et d'une valorisation sans précédent. Les outils nécessaires à l'analyse des corpus traditionnels (numérisation des originaux, métadonnées de transcription diplomatique, classements dynamiques, édition en ligne) sont en voie d'offrir à la recherche un environnement qui permettra bientôt de les convertir intégralement en matériau numérique et, dans un deuxième temps, de les traiter avec les mêmes outils que les archives électroniques d'aujourd'hui et de demain. Comment? Avec quels outils? Pour leur diffusion, avec les instruments d'encodage et d'édition en ligne XML/TEI d'un outil à vocation universelle qui permettra de respecter toutes les spécificités des archives de la genèse. Ce chantier est déjà bien engagé. Et pour l'étude systématique des contenus, grâce aux dispositifs d'analyse qui permettront de les modéliser en y appliquant des outils statistiques. Les quantités de données accessibles sont déjà considérables. Un corpus comme celui de Gustave Flaubert compte environ 50000 pièces qui contiennent en moyenne une centaine de phénomènes génétiques observables par page: 5 millions d'occurrences complexes. La moitié environ de ces occurrences a été analysée. Pour le seul périmètre des corpus étudiés à l'ITEM, on peut évaluer la masse potentielle du matériau expérimental disponible à environ 100 millions d'occurrences. L'enjeu que rend envisageable aujourd'hui la conversion des archives au tout numérique est de taille : faire entrer ces recherches dans la logique des algorithmes et des masses de données, changer d'échelle et passer au quantitatif. Il commencera alors à être envisageable de parler sérieusement d'une science du texte.

Voilà, du moins, la vision optimiste " tout en rose ", comme la dessinait Bouvard à travers sa représentation idyllique du futur à la fin du dernier roman de Flaubert, dans un chapitre d'ailleurs inachevé laissé à l'état de manuscrit de travail. Comme la vision de Bouvard, notre hypothèse d'une conservation intégrale des archives par le numérique 
n'est pas utopique - elle est réalisable -, mais elle n'est pas non plus assurée : toutes les conditions ne sont pas aujourd'hui réunies - c'est le moins qu'on puisse dire - pour que le scénario de «l'âge d'or" s'impose à notre avenir. Pour faire bonne mesure, passons maintenant à la vision pessimiste, «tout en noir », comme la formait Pécuchet à la fin du même roman. Il s'agit bien d'un film catastrophe dont le synopsis essentiel tient en deux cauchemars.

\section{Tout perdre}

Le premier cauchemar concerne la pérennité même des documents et de leurs copies. On sait que les papiers modernes, notamment depuis les procédures de fabrication industrielle à base de pâte de bois, c'est-à-dire massivement depuis la seconde moitié du XIX ${ }^{e}$ siècle, apogée du manuscrit et du livre, se trouvent chargés d'une acidité fatale aux fibres de cellulose qui les condamne à disparaittre physiquement dans les deux siècles qui suivent. Le compte à rebours est facile à faire. On sait également que les manuscrits sont plus menacés encore que les livres imprimés en raison des encres métalliques de leurs tracés qui ajoutent aux risques de la charge acide celui d'une oxydation de toutes les zones couvertes par l'écriture à la plume. Ce « slow fire » a beau être « slow », ses ravages commencent à menacer concrètement une partie du patrimoine écrit. Les bibliothèques qui en ont la charge désacidifient en autoclave les livres et stabilisent les documents autant qu'elles le peuvent, mais pour les autographes, particulièrement fragiles, le traitement est un défi technique, chaque opération prend du temps, le coût est considérable et, si l'on considère le problème dans son ensemble, il s'agit bien d'une course contre la montre qui est loin d'être gagnée. Des pans entiers du patrimoine autographe sont irréversiblement perdus, comme c'est le cas également pour des centaines de milliers d'ouvrages imprimés. Donc, on numérise. Mais quelle est la durée de vie garantie d'un disque dur? En termes d'engagement des fabricants, entre 2 et 5 ans, 10 dans le meilleur des cas: du papier acide au disque dur la précarité s'est multipliée en moyenne par $50 . .$. Et cela dans l'hypothèse irréaliste où aucun accident ne viendrait jamais s'ajouter au dépérissement attendu des supports. Dans les laboratoires de recherche stratégique, en informatique par exemple où l'on sait de quoi il retourne, les données sensibles, par mesure de sécurité, sont recopiées sur de nouveaux disques au minimum toutes les semaines et, pour les fondamentaux, tous les jours. Les bibliothèques patrimoniales, comme ces laboratoires, ont pris la mesure du problème et procèdent donc à des sauvegardes régulières pour assurer la pérennité des fichiers. Inutile d'en calculer le coût, évidemment exorbitant : il dépassera vite celui du stockage matériel des documents réels et ne peut donc, à terme, se traduire que par une sévère politique de restriction en matière d'enrichissements futurs et par des mesures draconiennes de "désherbage " pour le stock du déjà acquis.

Le deuxième cauchemar n'a pas l'ampleur du premier, mais ses conséquences ne sont pas moins dramatiques en termes de pertes irréparables. L'hypothèse d'un « siècle d'or » qui s'ouvrirait pour la génétique avec l'entrée dans l'ère du tout numérique repose sur le fait que l'ordinateur possède toutes les conditions requises pour mémoriser l'historique intégral des opérations qui ont eu lieu lors de la genèse de l'œuvre - recherche d'information, rédaction, versions successives, corrections, ratures, mises en pages -, qu'il s'agisse d'ailleurs d'un texte ou de tout autre artéfact visuel ou sonore. Les dispositifs requis pour cet archivage automatique des anciens fichiers sont 
structurellement envisageables et ne demanderaient pas d'énormes moyens logiciels pour être rendus opérationnels: une indexation fine avec sauvegarde paramétrée et systématique (par exemple sur disque périphérique) pourrait suffire. Le problème, c'est qu'à quelques très rares exceptions près, ces dispositifs n'équipent aucun de nos ordinateurs de travail : ils n'ont pas été prévus. Au contraire : ce qui est prévu pour la bonne marche de l'ordinateur, c'est le nettoyage par le vide. Conclusion : au-delà de la session de travail, l'historique est potentiellement promis à un effacement inévitable. Un disque dur peut voir disparaître tous ses fichiers non sauvegardés sous l'effet progressif de ses procédures de remise à jour, le "formatage de bas niveau" qui examine chaque cluster (unité minimale de stockage des fichiers) avec, en cas de besoin ou par simple mesure de précaution, vocation à le vider pour libérer de la place en faveur de l'inscription de nouveaux fichiers : sous cet effet, le contenu de chaque ancien cluster non sauvegardé peut se trouver effacé définitivement du disque, en même temps que les partitions qui lui assignait une place physique... Quand l'affaire est faite, même les plus fins limiers de la brigade des mœurs n'y peuvent plus rien.

Et inutile de se faire trop d'illusions sur les chances de récupérer les fichiers supprimés : selon la même logique, ils restent effectivement accessibles par le biais d'outils de récupération, mais seulement à titre provisoire, aussi longtemps que l'ordinateur n'aura pas décidé de recycler les clusters dans lesquels ces fichiers obsolètes ont été écrits primitivement. La durée de leur sursis est imprévisible; ils restent accessibles aux outils de récupération comme s'ils étaient pérennes, mais pour le système, en revanche, ils sont vierges : ils apparaissent comme parfaitement libres pour l'écriture de nouvelles données, ce qui ne manque pas d'arriver, tôt ou tard. Rien donc n'est assuré si l'utilisateur - ou un dispositif interne - ne choisit pas de faire volontairement des sauvegardes régulières qu'il indexe pour les conserver et les mettre à l'abri d'une élimination automatique. Or l'utilisateur (en l'occurrence le créateur) a autre chose à faire : créer ; quant au dispositif qui pourrait s'en charger pour lui, il n'existe pas.

Que doit-on en conclure? Qu'en matière d'archives de la création, nous sommes en train de vivre une sorte de catastrophe sans précédent : pour les vingt années qui viennent de s'écouler, au cours desquels la majorité des créateurs s'est progressivement convertie au tout numérique, il ne subsiste déjà plus aucun document de genèse, aucun brouillon, aucune trace génétique interprétable. Cette culture du tout numérique qui a réalisé le miracle de pouvoir virtuellement tout conserver aura in fine tout perdu : par négligence et par désintérêt, bien plus que par incompétence. Prenons la juste mesure du problème : il s'agit d'un trou archivistique total et irrémédiable qui n'a aucun équivalent dans l'histoire des trois derniers siècles, si ce n'est, peut-être, mais pour des durées plus courtes, les heures les plus sombres de notre histoire. Et ce trou déjà vieux de vingt ans continue à s'élargir chaque jour. C'est la totalité des archives de la création de notre temps qui passe à la trappe : pas seulement le travail des écrivains (ceux-là ont encore souvent la manie vintage de prendre au moins quelques notes sur des calepins), mais l'ensemble de notre production intellectuelle et artistique. Philosophes, historiens, architectes, compositeurs, chercheurs, cinéastes, etc.: du travail de toute cette génération, il ne restera donc à peu près rien en matière d'archives de la création. Quelques expériences institutionnelles d'archivage numérique, çà et là, ont bien été lancées, depuis quelques années, pour y remédier, mais seulement dans quelques secteurs sensibles (le "carnet de laboratoire", par exemple, au CNRS) et avec un succès limité. Dans d'autres métiers, les utilisateurs bricolent comme ils peuvent des dispositifs de 
fortune pour sauvegarder les archives dont ils ont besoin. Au total, rien qui soit à la mesure d'un problème qui concerne toutes les activités de création. Quand se décidera-ton à offrir à chaque ordinateur un équipement en série qui permette à chacun de récupérer l'historique de son travail aussi simplement que le permettait l'archivage des dossiers papier? En attendant, notre présent amnésique devient orphelin de son passé immédiat, et, au risque de nous perdre, nous avançons vers le futur en effaçant méthodiquement derrière nous les traces de nos propres pas. Pourquoi ?

\section{Communiquer ou transmettre}

Depuis trois siècles, les créateurs avaient multiplié les précautions et les efforts pour nous transmettre les traces de leur travail, avec l'espoir qu'à travers elles leurs œuvres seraient mieux comprises : en pensant aussi que le futur, notre présent, s'enrichirait par ce transfert d'expérience. Cette éthique de la transmission a vécu. Pour ce qui se crée aujourd'hui, pour le travail accompli par les créateurs contemporains, personne ne se soucie plus de savoir s'il en restera la moindre trace. D'où vient que notre temps soit tout à coup devenu à ce point indifférent à l'image de sa créativité, au sens de sa propre démarche? Négligence plus qu'incompétence puisqu'au même moment nous disposons des outils les plus performants qui aient jamais existé pour y parvenir : négligence, mais surtout désintérêt profond pour le transfert et la transmission, indifférence et même désinvolture à l'égard des cycles longs. Nous n'avons plus aucune foi réelle en l'avenir, ni dans la chaîne des temps, ni finalement dans l'enseignement du passé. Surtout pas de stock. Notre doxa mise tout sur le cycle court : vitesse du transfert, visibilité immédiate, notoriété maximale. Il est difficile de ne pas associer cette religion du flux tendu, cette ivresse de l'impact publicitaire, cette impatience du résultat et cette fuite en avant dans l'éphémère, avec la manière dont l'industrie des mass média et de l'informatique ont pu transfigurer notre environnement quotidien et notre axiologie: le tout numérique a accouché du tout communicationnel.

Culte de la communication en temps réel, abolition de l'espace, oubli généralisé du passé et du futur: inutile de se cacher derrière son petit doigt, sous couvert d'industrie culturelle, ce qui dicte ici sa loi c'est la nouvelle culture du tout industriel façon «nouvelles technologies ». Pas les contenus, mais les tuyaux: la seule industrie à haute valeur ajoutée qui assure des progressions annuelles à deux chiffres lorsque le reste a été mondialisé ailleurs, grâce au dumping social, à l'autre bout de la planète. Ce qui compte, au fond, c'est la bulle d'un présent ramené aux échéances des mandats, des appels d'offre et des générations de matériels, trois à quatre ans tout au plus : une bulle irisée de tous les espoirs de profits qui l'ont fait naître, mais par nature destinée à éclater - moyennant quelques dommages collatéraux - au profit d'une nouvelle bulle. Telle est la nouvelle hache à débiter l'Histoire en séquences brèves. Mais en pratique, au quotidien, c'est toute notre relation à la transmission qui se trouve rejetée du côté des vieilles lunes. Communiquer ou transmettre? Et si tout cela finalement n'était qu'une affaire de médium : silicium ou cellulose?

Notre temps a vu se construire la complémentarité mais désormais aussi la concurrence entre deux perceptions du texte et de l'image: l'une pragmatique et expéditive de consultation, d'information, d'indexation et de communication - celle de l'écran et d'Internet; l'autre lente, d'acquisition, de réflexion, de délibération, de rêverie, de délectation ou de plaisir - celle du papier. Les machines numériques avec lesquelles nous 
calculons, écrivons, dessinons, filmons, sont sans faiblesses et sans compassion. Toutes primitives qu'elles soient encore, elles n'ont déjà plus rien de commun avec les rythmes de notre pensée et de notre corps. Un dixième de seconde leur suffit pour lire in extenso Madame Bovary, là où il me faut au minimum vingt heures : je lis donc un million de fois plus lentement que la machine... ou même bien pire, le double, si je prends le temps de lire pour le plaisir, en savourant chaque phrase. Quant à écrire Madame Bovary ... Flaubert y a consacré cinq ans de sa vie : autant qu'il en a fallu pour déchiffrer et classer les 5000 pages de brouillons qui en portent témoignage.

31 C'est ainsi : nos yeux ne perçoivent du réel qu'une vingtaine d'images par seconde. Il y a dans la relation au papier un luxe de lenteur qui est celui de notre rythme biologique, et de notre mode d'intellection : il faut du temps pour réfléchir, juger, apprendre, apprécier et concevoir. Et il en faut encore beaucoup plus pour faire œuvre durable. Voilà un autre mot fétiche adoré par notre actualité : durable. Mais que veut dire durer? Peut-être le contraire, justement, de communiquer. Communiquer instantanément à travers l'espace est une performance utile et remarquable, certes : qui le nierait? Mais peut-être pas au point d'en oublier l'autre dimension, celle de la durée, sans laquelle l'idée même de transfert perd son sens. Faire un buz qui vous assure une notoriété planétaire en quelques jours est un miracle dont les créateurs ont toujours rêvé, au risque d'entraîner leur œuvre dans la forclusion de la mode et des cycles courts. Aussi vite oublié que vite célèbre : le temps se venge toujours de ce que l'on a voulu faire sans lui. Or, justement, prendre le temps d'élaborer un message ou une œuvre dignes d'être transmis non seulement à tous dans l'espace, mais aussi et surtout dans le temps, à travers la longue durée, à tous ceux qui sont à naître : n'est-ce pas cela, la vocation de ce que l'on appelait, naguère, la culture ? L'oublier peut avoir un prix redoutable. Andy Warhol, dès 1967, nous en avait prévenu: «À l'avenir, chacun aura son quart d'heure de célébrité. » Et, pour éviter toute ambiguïté, il ajoutait : «Oui, il y aura une époque, où chacun pourra dire ce qu'il pense... et en toute liberté, car alors tout le monde pensera la même chose. »

\section{NOTES}

1. Issue des liens entre le CNRS et la recherche universitaire, et réunissant des chercheurs d'horizons critiques très divers (psychanalyse, narratologie, sociologie, thématique, linguistique, etc.), la génétique des textes a pu se former en France grâce à un dispositif original qui a permis à plusieurs équipes de spécialistes (corpus Heine, Proust, Zola, Aragon, Flaubert, Valéry, Nerval, Joyce, Sartre, etc.) de se regrouper au sein d'une même structure du CNRS : le Centre d'Analyse des Manuscrits (1974-1981) transformé et élargi, depuis 1982, en Institut des Textes et Manuscrits Modernes (ITEM). Cet institut, lié par convention notamment à la BnF et à l'ENS (dont il est devenue une des UMR en 2001), s'est employé à développer des méthodes d'analyse spécifiques pour l'étude du manuscrit moderne et des archives de la création, en donnant naissance à une discipline nouvelle: la "génétique des textes et des formes ", qui rassemble aujourd'hui cent cinquante chercheurs répartis en vingt équipes. Leader dans son domaine, et reconnu à l'échelle internationale pour son rôle pilote et fédérateur, l'ITEM a poursuivi son développement par la création de nouveaux pôles de recherche (génétique des arts, écriture 
scientifique, thématiques multi-corpus, édition numérique des documents complexes, etc.) en s'associant à la diffusion de la discipline dans de nombreuses universités ou institutions de recherche françaises et étrangères.

2. À l'exception de quelques grandes entreprises philologiques à l'ancienne comme il en existe en Allemagne, ou de quelques grands éditeurs académiques comme Brépols, mais toujours dans le cadre d'opérations exceptionnelles.

3. Voir notre article, «Le cauchemar de Proust ", Médium, $\mathrm{n}^{\circ} 10$, Le Numérique en toutes lettres, 2007, p. 125-137.

\section{RÉSUMÉS}

La génétique des textes et des formes étudie les archives de la création pour interpréter les œuvres d'après leurs documents de genèse (plan, brouillons, notes, etc.). Ces millions de documents autographes nous ont été légués par les créateurs des trois derniers siècles comme la trace de leur travail. L'outil numérique contemporain des premières recherches de ce type s'est révélé indispensable pour traiter ces archives complexes et massives. À l'heure où les traces du travail créatif, dans tous les domaines, deviennent elles-mêmes numériques, peut-on s'attendre à un âge d'or de l'approche génétique dans la mesure où il est devenu possible de "tout conserver»? Ou à un cauchemar? Car la réalité est que, depuis vingt ans, les archives numériques de la création sont pour l'essentiel perdues, et continuent à se perdre, faute de support pérenne et de procédure de sauvegarde automatique des fichiers. Cette destruction irréversible des traces est sans précédent. Pourquoi notre présent est-il si désinvolte vis-à-vis de sa propre mémoire? Peut-être parce que le diktat du tout communicationnel et du flux tendu, qui exclut le différé et le stock, nous conduit à privilégier le court terme et la rentabilité immédiate, en oubliant la logique des cycles longs et de la transmission intergénérationnelle.

Textual genetics studies the archives of creation in order to interpret works according to their genetic documents (outlines, drafts, notes, etc.). These millions of autograph documents have been left by the creators of the last three centuries as a trace of their work. Digital tools, whose invention is contemporary of the early research of this type, have proved essential to handle these complex and massive archives. As traces of creative work in all fields become themselves digital, can we expect a golden age of the genetic approach since it has become possible to " preserve everything »? Or will it be a nightmare ? For, in fact, over the last twenty years, the digital archives of creation have essentially been lost, and continue to be lost because of a lack of sustainable recording media and automatic file backup process. This irreversible destruction of traces is unprecedented. Why then is our present so casual towards its own memory? Perhaps because the tyranny of the all-communicative and «just-in-time», which excludes the deferred and the stock, leads us to favor short term and immediate profitability, ignoring the logic of long cycle and intergenerational transmission. 
INDEX

Mots-clés : archives, manuscrits, génétique des textes, numérique, mémoire, transmission, communication, brouillons, processus, création, traces

Keywords : manuscripts, textual genetics, digital, memory, draft, process

\section{AUTEUR}

PIERRE-MARC DE BIASI

Directeur de l'Institut des textes et manuscrits modernes (CNRS-ENS, UMR 8132).

Parmi les publications :

"Le cauchemar de Proust », Médium, n 10, Le Numérique en toutes lettres, 2007, p. 125-137. 